\title{
Content-Based Image Retrieval (CBIR) in Big Histological Image Databases
}

Type of article: Conference abstract

Edwiges G. H. Grata ${ }^{1}$, Vania V. Estrela ${ }^{1}$, Ana C. B. Monteiro ${ }^{2}$, Reinaldo P. Franca ${ }^{2}$, Yuzo Iano $^{2}$, Abdeldjalil Khelassi ${ }^{3}$, Navid Razmjooy ${ }^{4}$, Maria A. de Jesus ${ }^{1}$

${ }^{1}$ Dep. of Telecommunications, Fluminense Federal University (UFF), RJ, Brazil

${ }^{2}$ School of Electrical and Computer Engineering (FEEC), UNICAMP, Campinas, SP, Brazil

${ }^{3}$ University of Tlemcen, Tlemcen, Algeria

${ }^{4}$ Department of Electrical Engineering, Tafresh University, Tafresh, Iran

Corresponding Author: vania.estrela.phd@ieee.org

\section{Abstract:}

Background: Automatic analysis of Histopathological Images (HIs) demands image processing and Computational Intelligence $(\mathrm{CI})$ techniques. Both Computer-Aided Diagnosis (CAD) and Content-Based Image-Retrieval (CBIR) systems assist diagnosis, disease discovery, and biological decision-making. Classical tests comprise screening examinations and biopsy. Histopathology slides offer more ample diagnosis data. However, manual examination of microscopic images is labor-intensive and time-consuming and may depend on a subjective assessment by the pathologist, which can be a challenge.

Methods: This work discusses a CBIR framework to extract and handle histological data, histological metadata, integrated patient records, specimen metadata, attributes, and similar stored files. This work presents a scalable image-retrieval framework for intelligent HI analysis with real-time retrieval. The potential applications of this framework include image-guided diagnosis, decision support, healthcare education, and efficient biological data management.

Results: The considerable amount of biological-related data prompted the development and deployment of large-scale databases and data-driven techniques to bridge the semantic gap between images and diagnostic information. The new cloud computing technologies and the concept of cyber-physical systems have improved the CBIR architectures considerably. The proposed scalable architecture relies on CI and validates performance on several HIs acquired from microscopic tissues. Extensive assessments show improvements in terms of disease classification and retrieval tests. Conclusion: This research effort significant contributions are twofold. 1) Defining a comprehensive and large-scale CBIR framework to analyze HIs with highdimensional features and CI methods successfully. 2) high-performance updating and optimization strategies improve the querying while better handling new training samples than traditional methods.

Keywords: Content-Based Image Retrieval (CBIR), High Dimensionality, Histopathological Image Analysis, Large Image Retrieval, Semantic Gap, Cloud Computing.

\section{Conflict of interest statement}

This article is a conference abstract presented at the International Congress on Health Sciences and Medical Technologies, Tlemcen Algeria 5-7 December 2019, ICHSMT'19. 
Medical Technologies Journal, Volume: 4, Issue: 3, July-September 2020, Pages:581-581. Doi :

https://doi.org/10.26415/2572-004X-vol4iss3p582-582

\section{Authors' biography}

No Biography.

\section{References}

No references 